\title{
Classical Roots of Knot Theory
}

\author{
JózEF H. PRZYTYCKI $\dagger$
}

Department of Mathematics, George Washington University, Washington, DC 20052, USA

\begin{abstract}
Vandermonde wrote in 1771: "Whatever the twists and turns of a system of threads in space, one can always obtain an expression for the calculation of its dimensions, but this expression will be of little use in practice. The craftsman who fashions a braid, a net, or some knots will be concerned, not with questions of measurement, but with those of position: what he sees there is the manner in which the threads are interlaced." We sketch in this essay the history of knot theory stressing the combinatorial aspect of the theory that is so visible in Jones type invariants. (C) 1998 Elsevier Science Ltd. All rights reserved
\end{abstract}

Next he [Alexander] marched into Pisidia where he subducd any resistance which he encountered, and then made himself master of Phrygia. When he captured Gordium [in March $333 \mathrm{BC}$ ] which is reputed to have been the home of the ancient king Midas, he saw the celebrated chariot which was fastened to its yoke by the bark of the cornel-tree, and heard the legend which was believed by all barbarians that the fates had decreed that the man who untied the knot was destined to become the ruler of the whole world. According to most writers, the fastenings were so elaborately intertwined and coiled upon one another that their ends were hidden: in consequence Alexander did not know what to do, and in the end loosened the knot by cutting through it with his sword, whereupon the many ends sprang into view. But according to Aristobulus he unfastened it quite easily by removing the pin which secured the yoke to the pole of the chariot, and then pulling out the yoke itself.Plutarch of Chareonera (c. AD 46-122), "Lives" ([1] p. 271).

The goal of this paper is to present the history of ideas which led up to the development of modern knot theory. We will try to be more detailed when pre-twentieth century history is reported. With more recent times we are more selective, stressing developments related to Jones type invariants of links. I am not a professional historian so my account is amateurish but I hope it can be treated as an invitation to more thorough exposition. $\neq$

Knots have fascinated people from the dawn of human history. We can wonder what caused a merchant living about $1700 \mathrm{BC}$ in Anatolia and exchanging goods with Mesopotamians to choose braids and knots as his seal sign, see Fig. 1. We can guess however that knots on seals or cylinders appeared before proper writing was invented about $3500 \mathrm{BC}$.

It is tempting to look for the origin of knot theory in Ancient Greek mathematics (if not earlier). There is some justification to do so: a Greek physician named Heraklas, who lived during the first century $\mathrm{AD}$ and who was likely a pupil or associate of Heliodorus wrote an essay on surgeon's slings. $\$$ Heraklas explains, giving step-by-step instructions, eighteen ways to tie orthopedic slings. His work survived because Oribasius of Pergamum (c. 325-400, physician of the emperor Julian the Apostate) included it toward the end of the fourth century in his "Medical Collections". The oldest extant manuscript of "Medical Collections" was made in the tenth century by the Byzantine physician Nicetas. The Codex of Nicetas was brought to Italy in the fifteenth century by an eminent Greek scholar, J. Lascaris, a refugee

$\uparrow$ Supported by USAF grant 1-443964-22502 while visiting the Mathematics Department, U.C. Berkeley.

†There are some books which treat the history of topics related to knot theory [2-5]. J. Stillwell's textbook [6] contains very interesting historical digressions.

§Heliodorus, who lived at the time of Trajan, also mentions in his work knots and loops [7]. 

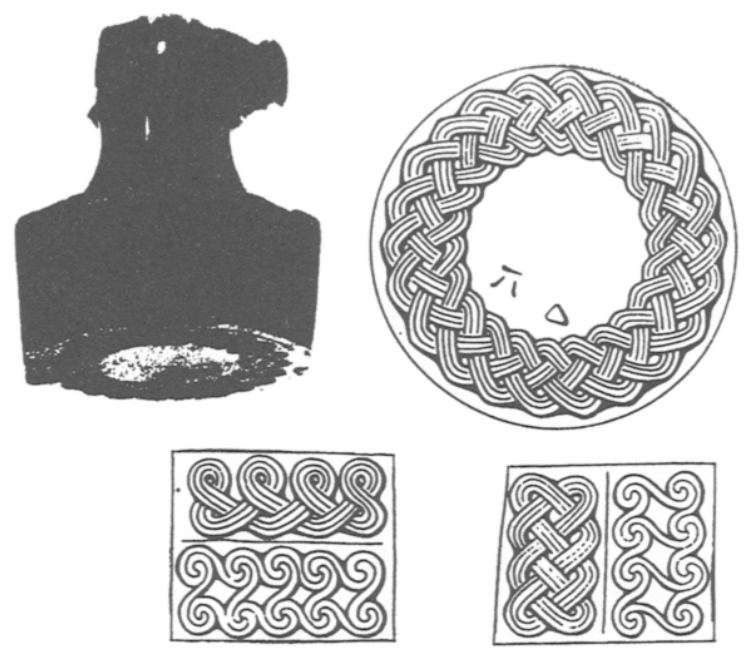

Fig. 1. Stamp seal, about $1700 \mathrm{BC}$ (in the British Museum). "On the octagonal base [of hammer-handled haematite seall are patterns surrounding a hieroglyphic inscription (largely erased). Four of the sides are blank and the other four are engraved with elaborate patterns typical of the period (and also popular in Syria) alternating with cult scenes..." [102], p. 93.

from Constantinople. Heraklas' part of the Codex of Nicetas has no illustrations, and around 1500 an anonymous artist depicted Heraklas' knots in one of the Greek manuscripts of Oribasus "Medical Collections". (In Fig. 2 we reproduce the drawing of the third Heraklas knot together with the original description of Heraklas.) Vidus Vidius (1500-1569), a Florentine who became physician to Francis I (King of France, 1515-1547) and professor of medicine in the Collège de France, translated the Codex of Nicetas into Latin; it contains also drawings of Heraklas' surgeon's slings by the Italian painter, sculptor and architect Francesco Primaticcio (1504-1570) [8,9].

Heraklas' essay should be taken seriously as far as knot theory is concerned. It is not knot

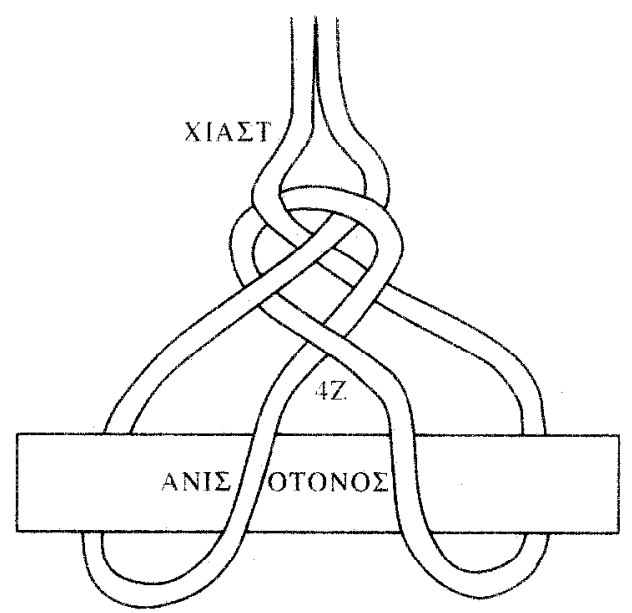

Fig. 2. The crossed noose. "For the tying the crossed nose, a cord, folded double, is procured, and the ends of the cord are held in the left hand, and the loop is held in the right hand. Then the loop is twisted so that the slack parts of the cord crossed. Hence the noose is called crossed. After the slack parts of the cord have been crossed, the loop is placed on the crossing, and the lower slack part of the cord is pulled up through the middle of the loop. thus the knot of the noose is in the middle, with a loop on one side and two ends on the other. This likewise, in function, is a noose of unequal tensions". [8] 
theory proper but rather its application. The story of the survival of Heraklas' work and efforts to reconstruct his knots in the Renaissance is typical of all science disciplines; and efforts to recover lost Greek books provided the important engine for the development of modern science. This was true in mathematics as well: the beginnings of modern calculus in the seventeenth century can be traced to efforts of reconstructing lost books of Archimedes and other ancient Greek mathematicians. It was only the work of Newton and Leibniz which went much farther than their Greek predecessors.

Gottfried Wilhelm Leibniz (1646-1716) speculated that aside from calculus and analytical geometry there should exist a "geometry of position" (geometria situs) which deals with relations depending on position alone (ignoring magnitudes). In a letter to Christian Huygens (1629-1695), written in 1679 [10], he declared:

I am not content with algebra, in that it yields neither the shortest proofs nor the most beautiful constructions of geometry. Consequently, in view of this, I consider that we need yet another kind of analysis, geometric or linear, which deals directly with position, as algebra deals with magnitude.

I do not know whether Leibniz had any convincing example of a problem belonging to the geometry of position. According to [11]:

As far back as 1679 , Leibniz, in his Characteristica Geometrica, tried to formulate basic geometric properties of geometrical figures, to use special symbols to represent them, and to combine these properties under operations so as to produce others. He called this study analysis situs or geometria situs ... To the extent that he was at all clear, Leibniz envisioned what we now call combinatorial topology.

The first convincing example of geometria situs was studied by Leonard Euler (1707-1783). This concerns the bridges on the River Pregel at Königsberg (then in East Prussia), shown in Fig. 3. Euler solved (and generalized) the bridges of Königsberg problem and on 26 August, 1735 presented his solution to the Russian Academy at St. Petersburg (it was published in 1736) [12]. With the Euler paper, graph theory and topology were born. Euler started his paper by remarking:

The branch of geometry that deals with magnitudes has been zealously studied throughout the past, but there is another branch that has been almost unknown up to now; Leibniz spoke of it first, calling it the "geometry of position" (geometria situs). This branch of geometry deals with relations dependent on position; it does not take

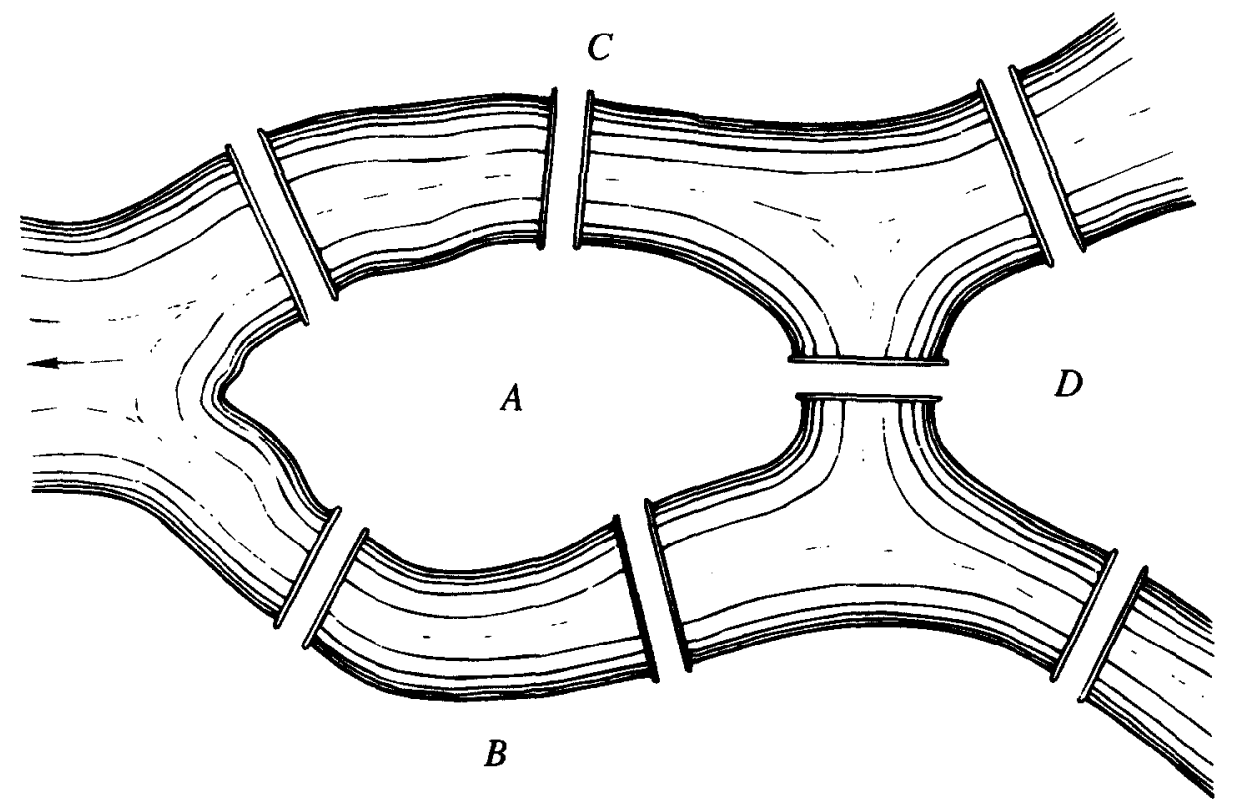

Fig. 3. Bridges of Königsberg. 

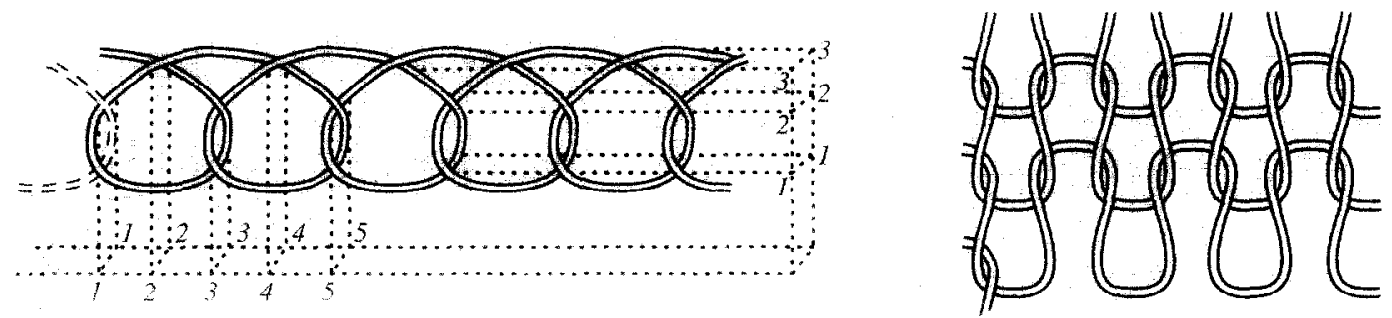

Fig. 4. Knots of Vandermonde.

magnitudes into consideration, nor does it involve calculation with quantities. But as yet no satisfactory definition has been given of the problems that belong to this geometry of position or of the method to be used in solving them.

For the birth of knot theory one had to wait another 35 years. In 1771, AlexandreTheophile Vandermonde (1735-1796) wrote the paper "Remarques sur les problèmes de situation" ("Remarks on problems of positions") where he specifically places braids and knots as a subject of the geometry of position [13], see Fig. 4. In the first paragraph of the paper Vandermonde wrote:

Whatever the twists and turns of a system of threads in space, one can always obtain an expression for the calculation of its dimensions, but this expression will be of little use in practice. The craftsman who fashions a braid, a net, or some knots will be concerned, not with questions of measurement, but with those of position: what he sees there is the manner in which the threads are interlaced.

In our search for the origin of knot theory, we arrive next at Carl Friedrich Gauss (1777-1855). According to $[14,15]$ :

One of the oldest notes by Gauss to be found among his papers is a sheet of paper with the date 1794. It bears the heading "A collection of knots" and contains thirteen neatly sketched views of knots with English names written beside them.. With it are two additional pieces of paper with sketches of knots. One is dated 1819; the other is much later.. t

In July of 1995 I finally visited the old library in Göttingen, I looked at knots from 1794; in fact not all of them are drawn, some are only described (see Fig. 5, for one of the drawings).

There are other fascinating drawings in Gauss' notebooks. There is, for example, the drawing of a braid (with analytic description at each height), shown in Fig. 6, with a note that

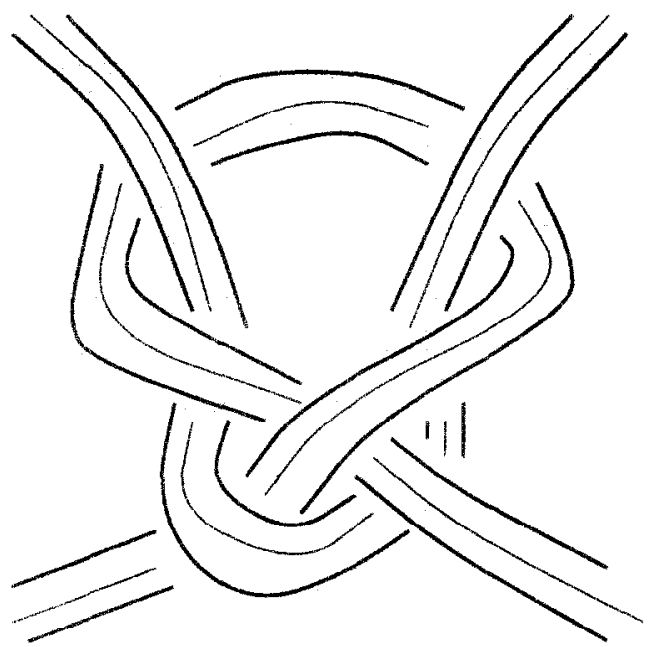

Fig. 5. Meshing knot, 10th knot of Gauss from 1794 


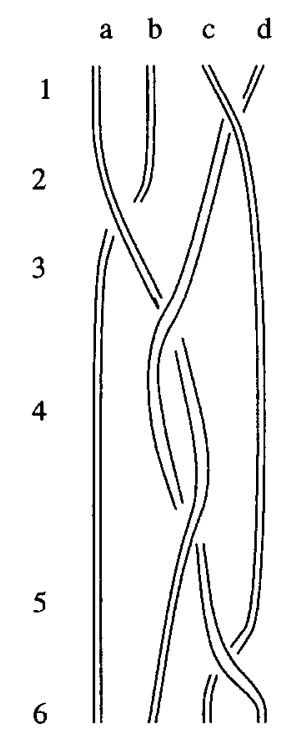

Veraindrungder Coordiniz

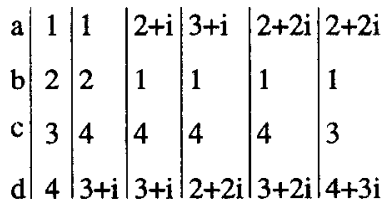

Fig. 6. "It is a good method of coding a knotting" (from a notebook of Gauss' (Handb. 7)).

it is a good method of coding a knotling. It is difficult to date the drawing; one can say for sure that it was done between 1814 and 1830 ; I would guess closer to 1814 . $\dagger$

In his note of 22 January 1833, Gauss introduces the linking number of two knots. $\ddagger$ Gauss' note presents the first deep incursion into knot theory; it establishes that the following two links are substantially different: $\bigcirc \bigcirc, 8$. Gauss' analytical method has been recently revitalized by Witten's approach to knot theory [18].

James Clerk Maxwell (1831-1879), in his fundamental book of 1873 "A treatise on electricity and magnetism" [19] writes: $§$

It was the discovery by Gauss of this very integral, expressing the work done on a magnetic pole while describing a closed curve in the presence of a closed electric current, and indicating the geometrical connection between the two closed curves, that led him to lament the small progress made in the Geometry of Position since the time of Leibnitz, Euler and Vandermonde. We have now, however, some progress to report chiefly due to Riemann, Helmholtz and Listing..

Maxwell goes on to describe two closed curves which cannot be separated but for which the value of the Gauss integral is equal to zero, see Fig. 7.

In $1876, O$. Boeddicker observed that, in a certain sense, the linking number is the number of the crossing points of the second curve with a surface bounded by the first curve [20,21]. Hermann Karl Brunn\| $[22,23]$ observed more than hundred years ago that the linking number of a two-component link, considered by Gauss, can be read from a diagram of the link.** If the link has components $K_{1}$ and $K_{2}$, we consider any diagram of the link and count each point at which $K_{1}$ crosses under $K_{2}$ as +1 for The sum of these, over all crossings of $K_{1}$ under $K_{2}$, is the Gauss linking number.

$\dagger$ As a curiosity, one can add that of one of the notebooks (Handb. 3) in which Gauss had also drawn some knot diagrams has braid motifs on its cover.

‡is method is analytical: the Gauss integral. In modern language, the Gauss integral computes analytically the degree of the map from a torus parameterizing a 2-component link to the unit 2-sphere.

$\S$ Gauss' note was first published in his collected works in 1867.

IGauss wrote in 1833, in the same note in which he introduced the linking number: "On the geometry of position, which Leibniz initiated and to which only two geometers, Euler and Vandermonde, have given a feeble glance, we know and possess, after a century and a half, very little more than nothing."

|Born 1 August 1862 in Rome, died 20 September 1939 in Munchen.

$* *$ It is also noted by Tait in 1877 , see p. 308 of [24]. 


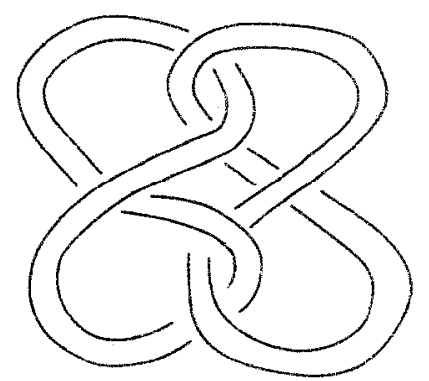

Fig. 7. The link of Maxwell

The year of 1847 was very important for knot theory (and for graph theory and topology as well). On one hand, Gustav Robert Kirchhoff (1824-1887) published his fundamental paper on electrical circuits [25]. It has deep connections with knot theory, but the relations were discovered only about a hundred years later (e.g. the Kirchhoff complexity of a circuit corresponds to the determinant of the knot or link determined by the circuit). On the other hand, Johann Benedict Listing (1808-1882), a student of Gauss, published his monograph "Vorstudien zur Topologie" [26]. A considerable part of the monograph is devoted to knots. $\dagger$ Listing stated in particular that the right-handed trefoil knot $\Theta$ and the left-handed trefoil $\rightleftharpoons$ are not equivalent. Later, Listing showed that the figure-eight knot $(B)$ and its mirror image $B$ are equivalent (we say that the figure-eight knot, also called the Listing knot, is amphicheiral).+

As we mentioned before, Maxwell, in his study of electricity and magnetism, had some thoughts on knots and links (in particular motivated by the freshly published Gauss' collected works). The origin of modern knot theory should be associated with four physicists: Hermann Von Helmholtz (1821-1894), William Thomson (Lord Kelvin) (1824-1907), Maxwell and Peter Guthrie Tait (1831-1901). (For Thomson's knots see Fig. 8.) We can quote after Tait's assistant in Edinburgh and later biographer, C. G. Knott [29]:
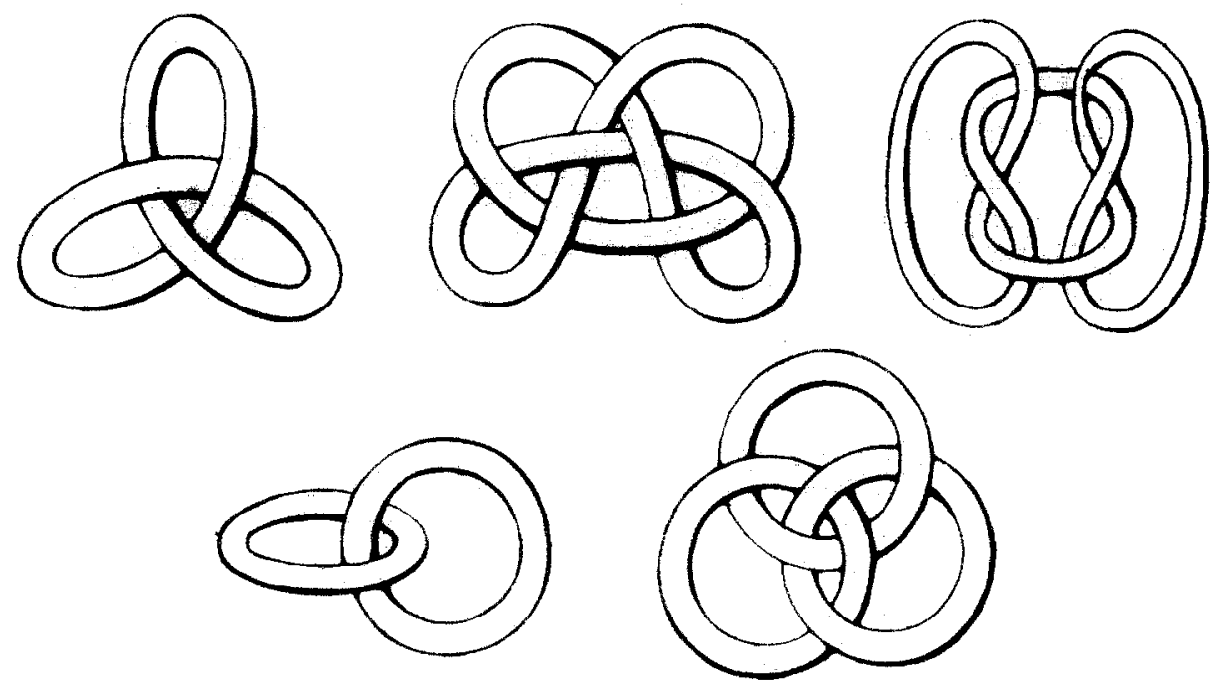

Fig. 8. Knots and links of William Thomson (Kelvin) from 1867

tEarlier, on 1 April 1836, Listing wrote a letter from Catania to "Herr Muller", with the heading "Topology". concerning... (2) winding paths of knots: and (3) paths in a lattice $[27,14]$.

tThis was observed in the note dated 18 March $1849[28]$. 
Tait was greatly impressed with Helmholtz's famous paper on vortex motion ([30]; 1858)... Early in 1867 he devised a simple but effective method of producing vortex smoke rings; and it was when viewing the behaviour of these in Tait's Class Room that Thomson was led to the conception of the vortex atom. In his first paper to the Royal Society of Edinburgh on February 18, 1867 [31], Sir William Thomson refers... to the genesis of the conception. In turn Thomson's theory was Tait's motivation to understand the structure of knots. In Tait's words: "I was led to the consideration of the form of knots by Sir W. Thomson's Theory of Vortex Atoms, and consequently the point of view which, at least at first, I adopted was that of classifying knots by the number of their crossings... The enormous number of lines in the spectra of certain elementary substances show that, if Thomson's suggestion be correct, the form of the corresponding vortex atoms cannot be regarded as very simple." [24]

There is an interesting letter from Maxwell to Tait dated 13 November 1867, which shows that Tait was sharing his ideas of knots with his friend [29,32]. In one of his rhymes [29] Maxwell wrote (clearly referring to Tait):

\author{
Clear your coil of kinkings \\ Into perfect plaiting, \\ Locking loops and linkings \\ Interpenetrating.
}

Tait describes his work on knots in the following words ([24], 1877):

\begin{abstract}
When I commenced my investigations I was altogether unaware that anything had been written (from a scientific point of view) about knots. No one in Section A at the British Association of 1876, when I read a little paper [33] on the subject, could give me any reference; and it was not till after I had sent my second paper to this Society that I obtained, in consequence of a hint from Professor Clerk-Maxwell, a copy of the very remarkable Essay by Listing, $\uparrow$ Vorstudien zur Topologie [26], of which (so far as it bears upon my present subject) I have given a full abstract in the Proceedings of the Society for Feb. 3, 1877. Here, as was to be expected, I found many of my results anticipated, but I also obtained one or two hints which, though of the briefest, have since been very useful to me. Listing does not enter upon the determination of the number of distinct forms of knots with a given number of intersections, in fact he gives only a very few forms as examples, and they are curiously enough confined to three, five and seven crossings only; but he makes several very suggestive remarks about the representation of a particular class of "reduced" knots... This work of Listing's and an acute remark made by Gauss (which with some comments on it by Clerk-Maxwell, will be referred to later), seem to be all of any consequence that has been as yet written on the subject.
\end{abstract}

\title{
Tait's paper was revised May 11, 1877; he finishes the paper as follows:
}

After the papers, of which the foregoing is a digest, had been read, I obtained from Professor Listing $\neq$ and Klein a few references to the literature of the subject of knots. It is very scanty, and has scarely any bearing upon the main question which I have treated above. Considering that Listing's Essay was published thirty years ago, and that it seems to be pretty well known in Germany, this is a curious fact. From Listing's letter (Proc. R.S.E. 1877, p. 316), it is clear that he has published only a small part of the results of his investigations. Klein himself [36] has made the very singular discovery that in space of four dimensions there cannot be knots.

The value of Gauss's integral has been discussed at considerable length by Boeddicker... in an Inaugural Dissertation, with the title Beitrag zur Theorie des Winkels, Göttingen, 1876.

An inaugural Dissertation by Weith, Topologishe Untersuchung der Kurven-Verschlingung, Zürich, 1876 [37], is professedly based on Listing's Essay. It contains a proof that there is an infinite number of different forms of knots! $\S$ The author points out what he (erroneously) supposes to be mistakes in Listing's Essay; and, in

†In 1883, Tait wrote [34] in Nature an obituary after Listing's death: "One of the few remaining links that still continued to connect our time with that in which Gauss had made Göttingen one of the chief intellectual centres of the civilised world has just been broken by the death of Listing... This paper [Vorstudien zur Topologie], which is throughout elementary, deserves careful translation into English..." After more than a hundred years the paper is not translated (only Tait's summary exists [35]) and one should repeat Tait's appeal again: The paper very much deserves translation. One can add that in 1932 the paper was translated into Russian.

$\ddagger$ The Library of the University of California has a copy of Vorstudien zur Topologie which Listing sent to Tait with the dedication.

$\$$ In fact it was proven only 20-30 years later and depended on the fundamental work of Poincaré on the foundation of algebraic topology. 

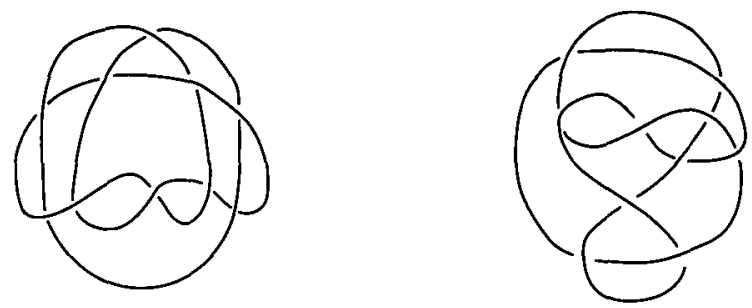

Fig. 9. Knots of Perko.

consequence, gives as something quite new an illustration of the obvious fact that there can be irreducible knots in which the crossings are not alternately over and under. $\dagger$ The rest of this paper is devoted to the relations of knots to Riemann's surfaces.

Tait, in collaboration with Reverend Thomas Penyngton Kirkman (1806-1895), and independently Charles Newton Little, $\$$ made considerable progress on the enumeration problem so that by 1900 there were in existence tables of (prime) knots up to ten crossings $[24,41,42,28]$. These tables were partially extended in Haseman's doctoral dissertation of $1916 \S$ [43]. Knots up to 11 crossings were enumerated by John H. Conway [44] before 1969. I

Knots up to 13 crossings were enumerated by C. H. Dowker and M. B. Thistlethwaite [47, 48], 1983.\|

To be able to make tables of knots, Tait introduced three basic principles (called now the Tait conjectures). All of them have been recently solved. The use of the Jones polynomial makes the solution of the first two Tait conjectures astonishingly easy [51-53] and the solution of the third Tait conjecture also uses essentially Jones type polynomials [54, 55]. We formulate these conjectures below:

T1. An alternating diagram with no nugatory crossings, of an alternating link realizes the minimal number of crossings among all diagrams representing the link. A nugatory crossing is drawn (defined) in Fig. 10(a).

(a)

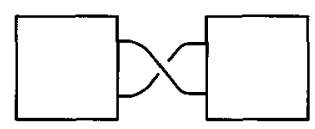

(b)

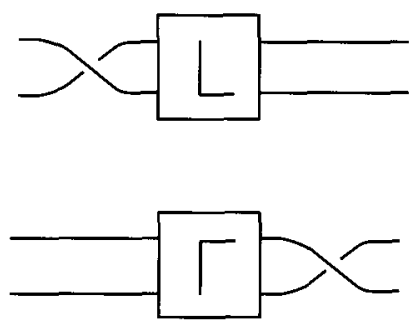

Fig. 10

$\dagger$ It was proven only in 1930 by Bankwitz [38], using the determinant of a knot.

†Born Madura India, May 19 1858. A.B. Nebraska 1879, A.M. 1884, Ph.D, Yale, 1885. Instructor math. and civil eng. Nebraska, 1880-84, assoc. prof. civil eng., 1884-1890, prof., 1890-1893; visited Göttingen and Berlin, 1898-1899, math. Stanford, 1893-1901, civil eng., Moscow, Idaho, from 1901, dean, col. eng., from 1911., died 31 August 1923 $[39,40]$.

\$Mary Gertrude Haseman was the fifth doctoral student of C. A. Scott at Brynn Mawr College.

IIK. A. Perko, a student of Fox at Princeton, and later a lawyer from New York, observed a duplication in the tables: two 10-crossing diagrams represented the same knot, see Fig. 9. Perko also corrected Conway's eleven crossing tables: 4 knots were missed $[45,46]$.

$\| \mathrm{J}$ im Hoste and Morwen Thistlethwaite have informed me that they have finished, working independently (Hoste with Jeff Weeks), a census of knots up to 16 crossings. They have found that there are 19536 prime alternating, 14 crossing knots, 85263 prime alternating, 15 crossing knots and 379799 prime alternating, 16 crossing knots. A census for prime nonalternating knots gives 27436 knots of 14 crossings, 168030 knots of 15 crossings and 1008906 knots of 16 crossings. The preliminary computation shows that there are less than 10 million prime 17 crossing knots. Knots and their mirror images are not counted separately $[49,50]$. 
T2. Two alternating diagrams, with no nugatory crossings, of the same oriented link have the same Tait (or writhe) number, i.e. the signed sum of all crossings of the diagram with the convention that is +1 and -1 is

T3. Two alternating diagrams, with no nugatory crossings, of the same link are related by a sequence of flypes (see Fig. 10(b)).

A very interesting survey on the developments in knot theory in the nineteenth century can be found in the Dehn and Heegaard article in the Mathematical Encyclopedia [56] of 1907.† In this context, the papers of Oscar Simony from Vienna $\ddagger$ are of great interest $[59,60]$. Figure 11 describes torus knots of Simony.

The fundamental problem in knot theory is to be able to distinguish nonequivalent knots. It was not achieved (even in the simple case of the unknot and the trefoil knot) until Jules Henri Poincaré (1854-1912) in his "Analysis Situs" paper [61] laid foundations for algebraic topology. Poul Heegaard (1871-1948) in his Copenhagen Dissertation [62] of 1898 constructed the 2 -fold branch cover of a trefoil knot and showed that it is the lens space $L(3,1)$, in modern terminology. He also showed that the analogous branch cover of the unknot is $S^{3}$. He distinguished $L(3,1)$ from $S^{3}$ using the Betti numbers; more precisely he showed that the first homology group is nontrivial and he clearly understood that it is a 3-torsion group. He did not state however that it can be used to distinguish the trefoil knot from the unknot, see p. 226 of [6].§ Heinrich Tietze (1880-1964) used in 1908 the fundamental group of the exterior of a knot in $R^{3}$, called the knot group, to distinguish the

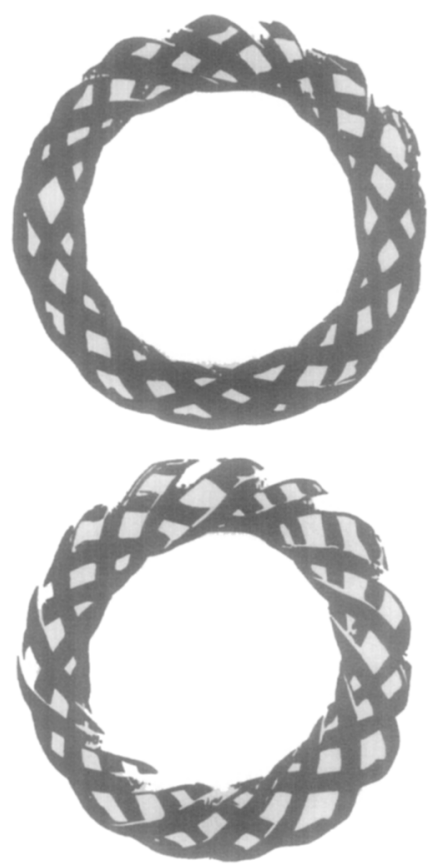

Fig. 11. Torus knots of Simony from 1884.

†According to Dehn's wife, Mrs. Toni Dehn, "Dehn and Heegaard met at the third International Congress of Mathematicians at Heidelberg in 1904 and took to each other immediately. They left Heidelberg on the same train, Dehn going to Hamburg and Heegaard returning to Copenhagen. They decided on the train that an Encyclopedia article on topology would be desirable, that they would propose themselves as authors to the editors, and that Heegaard would take care of literature whereas Dehn would outline a systematic approach which would lay the foundations of the discipline." [57].

łBorn 23 April 1852 in Vienna, died in 1915 [58].

\$For an English translation of the topological part of the Heegaard thesis, see the appendix to [63]. 

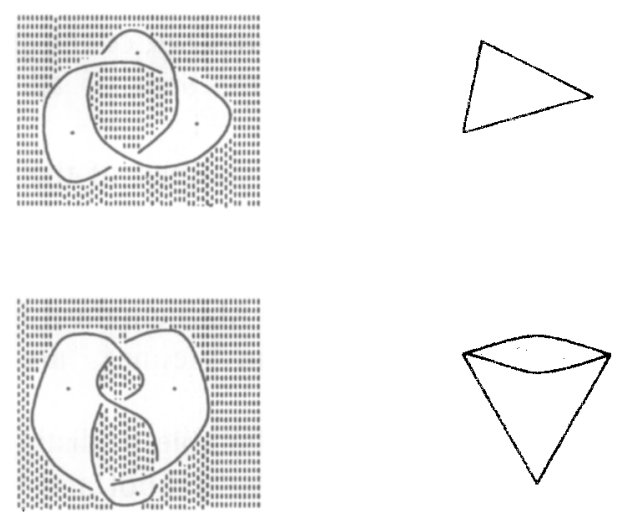

Fig. 12

unknot from the trefoil knot [64]. The fundamental group was essentiallyt introduced by Poincaré in his 1895 paper [61].

Wilhelm Wirtinger (1865-1945), in his lecture delivered at a meeting of the German Mathematical Society in 1905, outlined a method of finding a knot group presentation (now called the Wirtinger presentation of a knot group) [66]. Max Dehn (1878-1952), in his 1910 paper [67] refined the notion of the knot group and in 1914 was able to distinguish the righthanded trefoil knot $\Theta$ from its mirror image, the left-handed trefoil knot $\Theta$; that is, Dehn showed that the trefoil knot is not amphicheiral [68].

Tait was the first to notice the relation between knots and planar graphs. He colored the regions of the knot diagram alternately white and black (following $I$ isting) and constructed the graph by placing a vertex inside each white region, and then connecting vertices by edges going through the crossing points of the diagram (see Fig. 12) [56].

In 1912, George David Birkhoff (1884-1944), when trying to prove the four-color problem formulated in 1852 by Francis Guthrie (1831-1899), introduced the chromatic polynomial of a graph [70].

The breakthrough, from the point of view which focuses on the Jones type link invariants, was the invention by James Waddell Alexander (1888-1971) of a Laurent polynomial invariant of links [71]. Alexander was a colleague of Birkhoff and we can conjecture that he knew about the chromatic polynomial. $\$$

We know for sure that when W. T. Tutte was generalizing the chromatic polynomial [73] in 1947, he was motivated by the knot polynomial of Alexander. The Alexander polynomial can be derived from the group of the knot (or link). This point of view has been extensively developed since Alexander's discovery. More generally, the study of the fundamental group of a knot complement and the knot complement alone was the main topic of research in knot theory for the next fifty years, culminating in 1988 in the proof of Tietze's conjecture (that a knot is determined by its complement) [64] by Gordon and Luecke [74]. We can refer to the survey articles by Ralph Hartzler Fox (1913-1973) [75] and Gordon [76] or books [77-81] in

$\uparrow$ According to [3], Hurwitz' paper [65] of 1891 "may very well be interpreted as giving the first approach to the idea of a fundamental group of a space of arbitrarily many dimensions."

\$I 1978, W. Magnus wrote: "Today, it appears to be a hopeless task to assign priorities for the definition and the use of fundamental groups in the study of knots, particularly since Dehn had announced [69] one of the important results of his 1910 paper (the construction of Poincare spaces with the help of knots) already in 1907." [57]

$\$$ Birkhoff writes in [72]: “... Alexander, then [1911] a graduate student [at Princeton], began to be especially interested in the subject [analysis situs]. His well known 'duality theorem', his contributions to the theory of knots. and various other results, have made him a particularly important worker in the field". We can also mention that in the fall of 1909 Birkhoff became a member of the faculty at Princeton and lcft for Harvard in 1912. His 1912 paper $[70]$ ends with "Princeton University, May 4, 1912." 


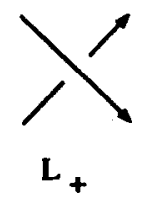

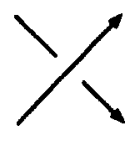

L.

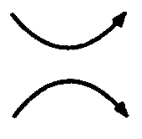

$\mathrm{L}_{0}$

Fig. 13

this respect. However, Alexander observed also that if three oriented links, $L_{+}, L_{-}$and $L_{0}$, have diagrams which are identical except near one crossing where they look as in Fig. 13, then their polynomials are linearly related [71]. An analogous discovery about the chromatic polynomial of graphs was made by Ronald M. Foster in 1932 (see [82]; compare also Formula (10) of [83]). In the early $1960 \mathrm{~s}$, J. Conway rediscovered Alexander's formula and normalized the Alexander polynomial $\Delta_{L}(t) \in Z\left[t^{ \pm 1 / 2}\right]$, defining it recursively [44] as follows:

(i) $\Delta_{o}(t)=1$, where $o$ denotes a knot isotopic to a simple circle,

(ii) $\Delta_{L_{+}}-\Delta_{L_{-}}=(\sqrt{t}-(1 / \sqrt{t})) \Delta_{L_{o}}$.

In the spring of 1984 , Vaughan Jones discovered his invariant of links $V_{L}(t)$ [84-86]. $\dagger$

Still in May of 1984 he was trying various substitutions to the variable $t$, in particular $t=-1$. He observed that $V_{L}(-1)$ is equal to the classical knots invariant, the determinant of a knot, but, initially, was unable to prove it. Soon he realized that his polynomial satisfies the local relation analogous to that discovered by Alexander and Conway and established the meaning of $t=-1$. Thus the Jones polynomial is defined recursively as follows:

(i) $V_{o}=1$,

(ii) $(1 / t) V_{L_{+}}(t)-t V_{L_{-}}(t)-(\sqrt{t}-(1 / \sqrt{t})) V_{L_{o}}(t)$.

In the summer and the fall of 1984, the Alexander and the Jones polynomials were generalized to the skein (named also Conway-Jones, Flypmoth, Homfly, Homflypt, generalized Jones, 2-variable Jones, Jones-Conway, Thomflyp, twisted Alexander) polynomial $P_{L} \in Z\left[a^{ \pm 1}, z^{ \pm 1}\right]$ of oriented links [88, 89]. This polynomial is defined recursively [88, 89] as follows:

(i) $P_{o}=1$,

(ii) $a P_{L_{+}}+a^{-1} P_{L_{-}}=z P_{L_{0}}$.

In particular $\Delta_{L}(t)=P_{L}(i, i(\sqrt{t}-(1 / \sqrt{t}))), V_{L}(t)=P_{L}\left(i t^{-1}, i(\sqrt{t}-(1 / \sqrt{t}))\right)$. In August 1985 , L. Kauffman found another approach to the Jones polynomial. It starts from an invariant $\langle\mathrm{D}\rangle \in Z[\mu, A, B]$ of an unoriented link diagram $D$ called the Kauffman bracket [53]. The Kauffman bracket is defined recursively by:

(i) $\langle o \ldots o\rangle=\mu_{i-1}$, where $o$ occurs $i$ times,

(ii) $\left\langle L_{+}\right\rangle=A\left\langle L_{0}\right\rangle+B\left\langle L_{\infty}\right\rangle$,

(iii) $\left\langle L_{-}\right\rangle=B\left\langle L_{0}\right\rangle+A\left\langle L_{\infty}\right\rangle$.

†Jones wrote in [87]: "It was a warm spring morning in May, 1984, when I took the uptown subway to Columbia University to meet with Joan S. Birman, a specialist in the theory of "braids"... In my work on von Neumann algebras, I had been astonished to discover expressions that bore a strong resemblance to the algebraic expression of certain topological relations among braids. I was hoping that the techniques I had been using would prove valuable in knot theory. Maybe I could even deduce some new facts about the Alexander polynomial. I went home somewhat depressed after a long day of discussions with Birman. It did not seem that my ideas were at all relevant to the Alexander polynomial or to anything else in knot theory. But one night the following week I found myself sitting up in bed and running off to do a few calculations. Success came with a much simpler approach than the one that 1 had been trying. I realized I had generated a polynomial invariant of knots".

$\ddagger$ The fact was also discovered independently in July 1984 by Lickorish and Millett.

\$At first, he thought that he had produced a new knot polynomial and only analyzing the polynomial realized that he had found a variant of the Jones polynomial. 


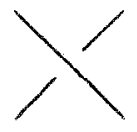

L.

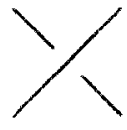

L

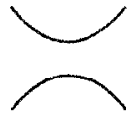

L 0

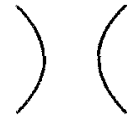

$\mathrm{L}_{\infty}$

Fig. 14

where $L_{+}, L_{\ldots}, L_{0}$ and $L_{\infty}$ denote four diagrams that are identical except near one crossing as shown in Fig. 14, and $\langle o \ldots o\rangle$ denotes a diagram of $i$ trivial components ( $i$ simple circles).

If we assign $B=A^{-1}$ and $\mu=-\left(A^{2}+A^{-2}\right)$ then the Kauffman bracket gives a variant of the Jones polynomial for oriented links. Namely, for $A=t^{-1 / 4}$ and $D$ an oriented diagram of $L$, we have

$$
V_{L}(t)=\left(-A^{3}\right)^{w(D)}\langle D\rangle,
$$

where $w(D)$ is the planar writhe (twist or Tait number) of $D$ equal to the algebraic sum of signs of crossings.

It should be noted, as observed first by Kauffman, that bracket \langle\rangle$_{\mu, A, B}$ is an isotopy invariant of alternating links (and their connected sums) under the assumption that the third Tait conjecture (recently proven by Menasco and Thistlethwaite $[54,55]$ ) holds.

In the summer of 1985 (two weeks before discovering the "bracket"), Kauffman invented another invariant of links [90], $F_{L}(a, z) \in Z\left[a^{ \pm 1}, z^{ \pm 1}\right]$, generalizing the polynomial discovered at the beginning of 1985 by Brandt, Lickorish, Millett and Ho [91, 92]. To define the Kauffman polynomial, we first introduce the polynomial invariant of link diagrams $\Lambda_{D}(a, z)$. It is defined recursively by:

(i) $\Lambda_{0}(a, z)=1$.

(ii) $\Lambda(a, z)=a \Lambda(a, z), \Lambda(a, z)=a^{-i} \Lambda_{1}(a, z)$,

(iii) $\Lambda_{D_{+}}(a, z)+\Lambda_{D}(a, z)=z\left(\Lambda_{\diamond}(a, z)+\Lambda_{D_{*}}(a, z)\right)$.

The Kauffman polynomial of oriented links is defined by $F_{L}(a, z)=a^{-\cdots(D)} \Lambda_{D}(a, z)$, where $D$ is any diagram of an oriented link $L$.

Jones type invariants lead to invariants of three-dimensional manifolds [93, 18, 94-99]. We mentioned already that Jones type invariants of knots have been used to solve Tait conjectures. The Jones discovery however not only introduced a delicate method of analyzing knots in 3-manifolds but related knot theory to other disciplines of mathematics and theoretical physics, such as statistical mechanics, quantum field theory, operator algebra, graph theory and computational complexity. On the other hand, the Jones polynomial gives a simple tool to recognize knots and as such is of great use for biologists (e.g. for the analysis of DNA) and chemists (see for example [100]).

\section{REFERENCES}

1. Plutarch of Chareonera, The Age of Alexander, translated by 1. Scott-Kilvert. Penguin Books, 1982.

2. Biggs, N. L.. Lloyd, E. K., and Wilson, R.J., Graph Theory 1736-1936. Clarendon Press, Oxford. 1986.

3. Chandler, B. and Magnus, W., The History of Combinatorial Group Theory: A Case Study in the History of Ideas. Studies in the History of Mathematics and Physical Sciences, Vol. 9, Springer, New York, 1982.

4. Crowe, M. J., A History of Vector Analysis: The Evolution of the Idea of a Vectorial System. Dover Publications. 1994 (first publ. 1967).

5. Dieudonné, J. A., A History of Algebraic and Differential Topology 1900-1960. Birkhäuser, Boston and Basel, 1989.

6. Stillwell. J., Classical Topology and Combinatorial Group Theory. Graduate Texts in Mathematics, Vol. 72. Springer, New York, 1980.

7. Sarton, G., Ancient Science through the Golden Age of Greece. Dover Publications, 1993 (first edition: Harvard University Press, 1952). 
8. Day, C. L., Quipus and Witches' Knots, With a Translation and Analysis of "Oribasius De Laqueis". University of Kansas Press, Lawrence, 1967.

9. Raeder, J., Oribassi Collectionum Medicarum Reliquiue. 4 vol., Leipzig and Berlin, 1928-1933.

10. Leibniz, G. W., Math. Schriften, 1 Abt,, Vol. 2, 1850, pp. 19-20.

11. Kline, M., Mathematical Thought from Ancient to Modern Times. Oxford University Press, 1972.

12. Euler, L., Solutio problematis ad geometriam situs pertinentis. Commentarii Academiae Scientiarum Imperialis Petropolitanae, 1736, 8, 128-140.

13. Vandermonde, A. T., Remarques sur les problèmes de situation, Mémoires de l'Académie Royale des Sciences (Paris), 1771, 566-574.

14. Stäckel, P., Gauss als Geometer, in the 10th volume of Gauss' collected works.

15. Dunnington, G. W., Carl Friedrich Gauss, Titan of Science. Hafner Publishing Co., New York, 1955.

16. van de Griend, P., Notes on Knots. Århus, 1993.

17. Falconer, W., A Universal Dictionary of the Marine. London, 1769.

18. Witten, E., Quantum field theory and the Jones polynomial. Comm. Math. Phys., 1989, 121, 351-399.

19. Maxwell, J. C., A Treatise on Electricity and Magnetism. Oxford, 1873.

20. Bocddicker, O., Bcitrag zur Theorien des Winkels, Dissertation, Göttingen, 1876.

21. Boeddicker, O., Erweiterung der Gausschen Theorie der Verschlingungen, Stuttgart, 1876.

22. Blaschke, W., Obituary: Hermann Brunn (in German). Jahresber. Deutsch. Math.-Verein., 1940, 50, $163-166$.

73. Brunn, H., Topologische Betrachtungen. Z. Math. Phys. 1892, 37, 106-116.

24. Tait, P. G., On knots I, II, III. In Scientific Papers. Cambridge University Press, 1898-1900. Including Trans. Roy. Soc. Edinburgh, 1877, 28, 35-79. Reprinted by Amphion Press, Washington, DC, 1993.

25. Kirchhoff, G. R., Über die Auflosung der Gleichungen, auf welche man bei der Untersuchung der linearen Vertheilung galvanischer strome geführt wird. Ann. Phys. Chem. 1847, 72, 497-508.

26. Listing, J. B., Vorstudien zur Topologie. Göttinger Studien (Abteilung 1), Vol. 1, 1847, pp. 811-875.

27. Breitenberger, E., Gauss und Listing: Topologie und Freundschaft (in German). Gauss-Ges. Göttingen Mitt., $1993,30,3-56$.

28. Little, C. N., Non-alternate \pm knots. Trans. Roy. Soc. Edinburgh, XXXIX, Part III (No. 30), for the session 1898-89 (read July 3, 1899), 771-778.

29. Knott, C. G., Life and Scientific Work of Peter Guthrie Tait, supplementing the two volumes of scientific papers published in 1898 and 1900. Cambridge University Press, 1911.

30. Helmholtz, H., Über Integrale der hydrodynamischen Gleichungen, Welche den Wirbelbewegungen entsprechen, Crelle's Journal für Mathematik, 1858, 55, 25-55; English translation by P. G. Tait, Philosophical Magazine, June 1867, 485-511.

31. Thomson, W. H., On vortex motion, Trans. Roy. Soc. Edinburgh, 1867, 25, 217-260. See also Proc. Roy. Soc. Edinburgh, February 1867.

32. Lomonaco, S. L., The modern legacies of Thomson's atomic vortex theory in classical electrodynamics. I'roc. Sympos. Appl. Math., 1996, 51, 145-166.

33. Tait, P. G., Some elementary properties of closed plane curves. Messenger of Mathematics, New Series, 69, 1877 (communicated at the 1876 meeting of the British Association). Reprinted in Scientific Papers of P. G. Tait, pp. $270-272$

34. Tait, P. G., Johann Benedict Listing. Nature, February 1, 1883. Reprinted in Scientific Papers of P. G. Tait.

35. Tait, P. G., Listing's Topologie (Introductory address to the Edinburgh Mathematical Society, November 9, 1883) Philosophical Magazine, January, 1884. Reprinted in Scientific Papers of P. G. Tait.

36. Klein, F., Mathematische Annalen, IX, 478.

37. Weith, H., Topologische Untersuchung der Kurven-Verschlingung. Inaugural dissertation, Zürich, 1876.

38. Bankwitz, C., Über die Torsionszahlen der alternierenden Knoten. Math. Ann., 1930, 103, $145-161$.

39. Catell, J. McKeen Birmhall, Dean R. Garrison (eds), American Men of Science, 3rd edn. The Science Press, New York, 1921.

40. Yale University, Obituary record of graduates deceased during the year ending July 1, 1924, New Haven (published by the University), 1924, p. 1211.

41. Kirkman, T. P., The enumeration description and construction of knots with fewer than 10 crossing. Trans. Roy. Soc: Edinburgh 1885, 32, 281-309.

42. Little, C. N., On knots, with a census for order 10. Trans. Connecticut Acad. Sci., 1885, 18, 374-378; also Ph.D. thesis, Yale University, 1885 (see Ref. [101]).

43. Haseman, M. G., On knots, with a census of the amphicheirals with twelve crossings. Trans. Roy. Soc. Edinburgh, 1918, 52, 235-255; also Ph.D. thesis, Bryn Mawr College, 1918 (see Ref. [101]).

44. Conway, J. H., An enumeration of knots and links. In Computational Problems in Abstract Algebra (ed. J. Leech). Pergamon Press, Oxford, 1969, pp. 329-358.

45. Perko, K. A. Jr., On the classification of knots. Proc. Amer. Math. Soc., 1974, 45, 262-266.

46. Perko, K. A., Primality of certain knots. Topology Proc., 1982, 7, 109-118.

47. Dowker, C. H. and Thistlethwaite, M. B., Classification of knot projections. Topology Appl., 1983, 16, 19-31.

48. 'I'histlethwaite, M. B., Knot tabulations and related topics. In Aspects of Topology, Lecture Notes Series, Vol. 93 , London Mathematical Society, 1985, pp. 1-76.

49. Hoste, J., e-mails, 2 and 7 September 1993, and 9 June 1994.

50. Thistlethwaite, M. B., e-mail, 16 February 1997.

51. Murasugi, K., Jones' polynomials and classical conjectures in knot theory. Topology, 1987, 26, 187-194. 
52. Thistlethwaite, M. B., A spanning tree expansion of the Jones polynomial. Topology, 1987. 26, 297-309.

53. Kauffman, L., State models and the Jones polynomial. Topology, 1987, 26, 395-407.

54. Menasco. W. M. and Thistlethwaite, M. B. The Tait flyping conjecture. Bull. Amer. Math. Soc., 1991, 25, 2 403-412

55. Menasco. W. M. and Thistlethwaite, M. B., The classification of alternating links. Ann. of Math., $1993,138$. 113.171

56. Dehn, M. and Heegaard, P., Analysid situs. Encykl. Math. Wiss., Vol. III, AB3, Leipzig, 1907, pp. 153-220.

57. Magnus, W. Max Dehn. Math. Intelligencer, 1978, 1, 132-143; also in Wilhelm Magnus Collected Papers, ed. G. Baumslag and B. Chandler. Springer, 1984

58. Poggendorff's Biographisch Literarisches Handwörterbuch, Vol. 3, 1858-83 (A-Z), 1898.

59. Simony, O., Uber eine Reihe neuer Thatsachen aus dem Gebiete der Topologie. Math. Ann., 1882, 19, 110-120; and $1884,24,253-280$.

60. Tietze, H. Über Simony-Knoten und Simony-Ketten mit vorgeschriebenen singulären Primzahlen für die Figur und für ihr Spiegelbild. Math. Z., 1943, 49, 351-369.

6L. Poincaré. H., Analysis Situs (\&12). Joumal d'Ecole Polytechnique Normale, 1895, 1, 1-121.

62. Heegaard, P., Forstudier til en Topologisk Teori for de algebraiske Fladers Sammenhang, København, 1898. Filosofiske Doktorgrad; French translation: Sur l'Analysis situs, Bull. Soc. Math. France, 1916, 44, 161-242.

63. Pryytycki, J. H., Knot Theory from Vandermonde to Jones, with the translation of the topological part of Poul Heegaard Dissertation: Forstudier til en topologisk teori for de algebraiske fladers sammenh[ae]ng, København 1898. Filosofisk Doktorgrad (Preliminary studies towards the topological theory of connectivity of algebraic surfaces), by A.H. Przybyszewska, Preprint 43, Odense University, 1993.

64. Tietze, H., Uber die topologischen Invarianten mehrdimensionaler Mannigfaltigkeitell. Monatsh. Math. Phys. 1908, 19, 1-118.

65. Hurwitz, A., Über Riemannsche Flächen mit gegebenen Verzweigung spunkten. Math. Ann. 1891, 39, 1-61.

66. Wirtinger, W., Über die Verzweigungen bei Funktionen von zwei Veränderlichen, Jahresber. Deutsch. Math. Verein., $1905,14,517$. (The title of the talk supposedly given on 26 September 1905 at the annual meeting of the German Mathematical Society in Meran.)

67. Dehn. M., Über die Topologie des dreidimensionalen Raumes. Math. Ann., 1910, 69, 137-168.

68. Dehn, M., Die beiden Kleeblattschlingen. Math. Ann., 1914, 75, 1-12.

69. Dehn, M., Jahresber. Deutsch. Math.-Verein., 1907, 16, 573

70. Birkhoff, G. D. A determinant formula for the number of ways of coloring a map. Ann. of Math., 1912, 2, 14 $42-46$.

71. Alexander, J. W., Topological invariants of knots and links. Trans. Amer. Math. Soc., 1928, 30, $275-306$.

72. Birkhoff, G. D., Fifty years of American Mathematics, Amer. Math. Soc. Semicentennial Addresses, Vol. 2, 1938 , 270-315. Also in George David Birkhoff Collected Mathematical Papers, Vol. 3. American Mathematical Society. 1950 , pp. 606-651.

73. Tutte, W. T., A ring in graph theory. Proc. Cambridge Phil. Soc, 1947, 43, 26-40.

74. Gordon. C. M. and Luecke, I. Knots are determined by their complements Bull. Amer. Math. Soc., 1989, 20, 83.87

75. Fox, R. H., A quick trip through knot theory. In Top. 3-manifolds, Proc. 1961 Top. Inst. Univ. Georgia, ed. M. K. Fort, Jr. Prentice-IIall, Englewood Cliffs, NJ, 1962, pp. 120-167.

76. Gordon, C. McA.. Some aspects of classical knot theory. In Knot Theory, L.N.M. Vol. 685, 1978, pp. 1-60.

77. Birman, J. S., Braids, Links and Mapping Class Groups. Ann. Math. Studies, Vol. 82. Princeton Univ. Press, Princeton, NJ, 1974.

78. Burde, G. and Zieschang, H., Knots. De Gruyter, 1985.

79. Kauffman, L., On Knots. Princeton University Press, 1987.

80. Reidemeister. K., Knotentheorie. Ergebn. Math. Grenzgeb. Bd.1. Springer. Berlin, 1932: English translation: Knot Theory. BSC Associates, Moscow, Idaho, 1983.

81. Rolfsen, D., Knots and Links. Publish or Perish. 1976.

82. Whitney, H., The coloring of graphs. Ann. of Math., 1932, 33, 688-718.

83. Birkhoff, G. D., On the polynomial expressions for the number of ways of coloring a map, Ann. Scuola Norm. Sup. Pisa (2). 1934, 3, 85-104. Also in George David Birkhoff Collected Mathematical Papers, Vol. 3, American Mathematical Society, 1950 , pp. 29-47.

84. Jones, V., Letter to J. Birman, May 1984.

85. Jones, $V_{\text {. }}$ A polynomial invariant for knots via Von Neumann algebras. Bull. Amer. Math. Soc, 1985,12, 103-111.

86. Jones, $V_{\text {. }}$ Hecke algebra representations of braid groups and link polynomials, Ann. of Math., 1987, 335-388.

87. Jones, V., Knot theory and statistical mechanics. Scientific American, 1990, 262, 11 98-103.

88. Freyd, P., Yetter, D., Hoste, J., Lickorish, W. B. R., Millett, K. and Ocneanu, A. A new polynomial invariant of knots and links. Bull. Amer. Math. Soc, 1985, 12, 239-249.

89. Przytycki, J. H. and Traczyk, P., Invariants of links of Conway type. Kobe J. Math., 1987, 4, 115-139.

90. Kauffman, L., An invariant of regular isotopy. Trans. Amer. Math. Soc., 1990, 318, $2417-471$.

91. Brandt, R. D., Lickorish, W. B. R. and Millett, K. C. A polynomial invariant for unoricnted knots and links. Invent. Math. 1986, 84, 563-573 
92. Ho, C. F., A new polynomial for knots and links; preliminary report. Abstracts Amer. Math. Soc., 1985, 6, 4 300

93. Atiyah, M., The Geometry and Physics of Knots. Cambridge University Press, 1990, p. 75.

94. Przytycki, J. H., Skein modules of 3-manifolds. Bull. Polish Acad. Sci., 1991, 39, 1/2 91-100.

95. Turaev, V. G., The Conway and Kauffman modules of the solid torus. Zap. Nauchn. Sem. Lomi, 1988, 167, 79-89. English translation in J. Soviet Math.

96. Reshetikhin, N. Y. and Turaev, V., Invariants of three manifolds via link polynomials and quantum groups. Invent. Math., 1991, 103, 547-597.

97. Turaev, V. G. and Viro, O. Y., State sum invariants of 3-manifolds and quantum 6j-symbols. Topology, 1992, 31, $865-902$.

98. Turaev, V. G. and Wenzl, H., Quantum invariants of 3-manifolds associated with classical simple Lie algebras. Internat. J. Math., 1993, 4, 2 323-358.

99. Przytycki, J. H., Algebraic topology based on knots: an introduction. In Proceedings of Knots 96, ed. Shin'ichi Suzuki. World Scientific Publishing Co., 1997, pp. 279-297.

100. Sumners, D. W., (ed.), Cozzarelli, N. R., Kauffman, L. H., Simon, J., Sumners, D.W., White, J. H. and Whittington, S. G., New Scientific Applications of Geometry and Topology. Proceedings of Symposia in Applied Mathematics, Vol. 45, American Mathematical Society, 1992.

101. Comprehensive Dissertation Index, 1861-1972, Mathematics and Statistics, Vol. 5. Xerox University Microfilms, Ann Arbor, Michigan, 1973. 